\title{
Diabète de type 1 et environnement
}

\section{Danièle Dubois-Laforgue José Timsit}

D. Dubois-Laforgue : Unité de diabétologie adulte, Service d'immunologie clinique, Hôpital Necker, 149, rue de Sèvres, 75015 Paris et Inserm U.342, Hôpital Saint-Vincent-de-Paul, avenue Denfert-Rochereau, 75014 Paris, France.

J. Timsit : Unité de diabétologie adulte, Service d'immunologie clinique, Hôpital Necker, Paris, France.
Le diabète de type 1 est une maladie auto-immune dirigée contre les cellules $\beta$ pancréatiques survenant sur un terrain génétique de susceptibilité. Les mécanismes du déclenchement de la maladie auto-immune ne sont pas connus mais semblent pouvoir intervenir très tôt dans la vie. La survenue de I'hyperglycémie est précédée $d^{\prime}$ une longue phase préclinique. L'absence de concordance complète pour la maladie chez les jumeaux monozygotes et I'augmentation rapide de I'incidence du diabète de type 1 suggèrent que des facteurs d'environnement peuvent moduler les stades initiaux et l'évolution de la maladie autoimmune.

\section{TIRÉS À PART}

J. Timsit. e diabète de type 1 est la conséquence d'une carence de I'insulinosécrétion due à la destruction spécifique des cellules $\beta$ des îlots de Langerhans par un processus auto-immun $\left(\mathrm{m} / \mathrm{s} 1993, n^{\circ} 10, p .1137\right)$. Le taux de concordance pour le diabète de type 1, deux à quatre fois plus élevé chez les jumeaux monozygotes que chez les jumeaux dizygotes, indique le rôle des facteurs génétiques dans le développement de la maladie.

\section{Physiopathologie et données épidémiologiques}

Le diabète de type 1 est une maladie polygénique avec un locus majeur situé dans la région HLA de classe II (gènes $D R$ et $D Q$ ) qui rend compte d'environ $40 \%$ de la susceptibilité; de nombreux autres locus ont été suggérés mais non confirmés. Chez I'homme, comme dans les modèles animaux de diabète de type 1 spontané - rat Bio Breeding (BB) et souris non obese diabetic (NOD) - le stade clinique de la maladie (hyperglycémie chronique) est précédé d'une longue phase au cours de laquelle des marqueurs d'activation du sys- tème immunitaire, autoanticorps et lymphocytes T autoréactifs, sont présents. Une insulite, infiltrat des îlots de Langerhans par des cellules mononucléées, précède la survenue du diabète. II est classiquement admis que I'activation du système immunitaire fait suite à une rupture de tolérance vis-à-vis d'auto-antigènes insulaires, conduisant à l'activation de lymphocytes Th1, mais sa cause n'est pas connue. Chez la souris NOD, au cours de la phase pré-clinique, cette réponse se diversifie à la fois vis-à-vis d'épitopes différents du même antigène et $d^{\prime}$ antigènes différents [1]. Des données similaires ont été observées chez les enfants nés d'un parent diabétique et qui évoluent vers le diabète de type 1 .

Chez I'homme, le rôle de l'environnement dans la physiopathologie de la maladie a été évoqué sur des arguments indirects. Le taux de concordance pour la maladie clinique chez les jumeaux monozygotes n'est que de $30 \%$ à $70 \%$ [2]. Il existe de fortes variations géographiques de I'incidence annuelle du diabète de type 1 : en Europe, I'incidence varie d'un facteur 10 (de 3,2/100000 en Macédoine à 41/100000 en Finlande) [3] et un gradient décroissant Nord-Sud a 
été décrit. L'incidence de la maladie augmente chez les migrants d'une région à faible incidence vers une zone de forte incidence [4]. Des "épidémies » saisonnières et l'agrégation de nouveaux cas dans des aires géographiques restreintes ont été rapportées. Surtout l'incidence annuelle du diabète de type 1 augmente considérablement en Europe, à un rythme très variable d'un pays à l'autre, en moyenne de 3,4\% par an [3]. En Finlande, pays où l'incidence annuelle est la plus élevée, I'incidence du diabète de type 1 a quadruplé en 40 ans. Cette augmentation concerne particulièrement les enfants de moins de 5 ans et ne peut s'expliquer par des facteurs génétiques.

Cependant, I'interprétation de ces données n'est ni simple ni probablement univoque. Chez I'homme, le diabète de type 1 est une affection dont l'expression clinique est hétérogène en ce qui concerne la grande variabilité de l'âge de survenue de I'hyperglycémie, son association éventuelle à d'autres affections autoimmunes spécifiques d'organe, et son caractère familial ou beaucoup plus souvent sporadique (90\% des cas). Les facteurs qui déterminent cette hétérogénéité ne sont pas connus. La phase préclinique du diabète de type 1 peut être très longue, jusqu'à plus de 30 ans. Une proportion élevée de jumeaux considérés comme discordants a en fait des auto-anticorps anti-cellules d'îlots et dans la population générale la prévalence des marqueurs d'auto-immunité anticellules d'îlots est supérieure à celle du diabète de type 1 , ce qui suggère l'existence de formes de la maladie auto-immune non ou très lentement évolutives. De même, chez la souris NOD, dans un même élevage, $60 \%$ des femelles et moins de $30 \%$ des mâles développent un diabète alors que tous les animaux présentent des signes histologiques d'insulite. Dans ce contexte, les facteurs d'environnement pourraient intervenir de façon précoce au stade du déclenchement du processus auto-immun pour le favoriser ou au contraire le bloquer, ou plus tard, lorsque la maladie autoimmune est engagée pour en moduler, positivement ou négativement, I'agressivité. De plus, à un stade tardif de la réduction de la masse de cellules $\beta$, des facteurs augmentant les besoins en insuline via une insulinorésistance, comme par exemple une infection, peuvent précipiter la survenue de I'hyperglycémie.

L'histoire naturelle du diabète de type 1 est mal connue. L'étude prospective des enfants nés d'un parent diabétique (situation qui correspond à moins de $5 \%$ des cas de diabète) a montré que l'apparition des premiers auto-anticorps (le plus souvent anticorps anti-insuline) est précoce, dans les deux premières années de vie [5]. Dans ce contexte, I'apparition des auto-anticorps est particulièrement fréquente chez les enfants porteurs des génotypes $D R B 1^{*} 03 / 04$ et 04/04. De même, chez les germains de patients atteints de diabète de type 1 , I'acquisition des auto-anticorps, qui est prédictive de l'évolution ultérieure vers le diabète, est précoce, le taux de "séroconversion » étant très faible après l'âge de 5 à 7 ans. Ces données suggèrent que si un agent de l'environnement est impliqué dans le déclenchement de la maladie autoimmune son intervention est très précoce, dès la vie foetale ou la petite enfance. Elles ont de fortes implications pour l'élaboration de stratégies de prévention de la maladie. Cependant, il n'est pas certain que ces observations puissent être extrapolées aux cas sporadiques qui sont majoritaires. La cinétique de la maladie auto-immune et de la destruction des cellules $\beta$ est variable. Les études familiales prospectives ont établi le fort caractère prédictif de la présence, chez les apparentés du premier degré de patients atteints de diabète de type 1 , des auto-anticorps anti-cellules d'îlot (ICA), anti-GAD (décarboxylase de I'acide glutamique), anti-IA-2 (tyrosine phosphatase) et anti-insuline, particulièrement chez les sujets porteurs d'anticorps multiples. Cependant, chez ces sujets, il n'est pas possible de déterminer la vitesse d'évolution vers le diabète de type 1 sur la base des marqueurs immunologiques ou génétiques actuellement disponibles [6]. Des facteurs d'environnement pourraient donc moduler cette évolution.

\section{La piste des agents infectieux}

Les agents infectieux, en particulier de nombreux virus, ont depuis long- temps été incriminés dans la physiopathologie du diabète de type 1 . Les observations de diabète aigu succédant à une destruction des cellules $\beta$ par une infection cytopathogène restent exceptionnelles. Un authentique diabète auto-immun est observé dans $10 \%$ à $20 \%$ des cas de rubéole congénitale, dans un délai de 5 à 25 ans. Une infection du pancréas par le virus a été montrée chez certains des sujets atteints. La survenue du diabète pourrait être secondaire à l'effet cytopathogène direct du virus pour les cellules $\beta$ et à un mimétisme moléculaire entre la protéine de capside et un antigène $\beta$-pancréatique de 52 kDa non encore identifié. Le lien entre infection virale et diabète de type 1 repose surtout sur des arguments épidémiologiques. En GrandeBretagne et en Suède, la distribution des naissances des enfants diabétiques est relativement restreinte en terme de date et de lieu, ce qui suggère le rôle potentiel d'infections périnatales [7]. Plusieurs études ont rapporté une association entre infections par entérovirus, plus particulièrement le virus coxsackie $B$ et la survenue ultérieure $d^{\prime} u n$ diabète de type 1 [8]. Les infections à entérovirus au cours de la grossesse pourraient constituer un facteur de risque de diabète de type 1 chez l'enfant. En Suède, des IgM anti-entérovirus et/ou I'ARN viral ont été recherchés au cours du premier trimestre dans le sérum de 85 femmes dont l'enfant a développé un diabète de type 1 avant l'âge de 15 ans et de 172 femmes dont les enfants n'ont pas développé la maladie. Le risque relatif de diabète de type 1 associé à ces marqueurs d'infection est de 13 [9]. En Finlande, où I'incidence du diabète de type 1 a augmenté, la prévalence des IgG anti-entérovirus (en particulier coxsackie) chez les femmes enceintes de la population générale a diminué, ce qui pourrait augmenter le risque $d^{\prime}$ infection chez les femmes et leurs enfants [10]. Chez les sujets récemment diabétiques, les études de prévalence des marqueurs sérologiques $d$ 'infection par entérovirus sont contradictoires ou d'interprétation difficile pour des raisons méthodologiques. Cependant, I'ARN du virus coxsackie est détecté plus fréquemment dans le plasma des enfants présentant un diabète de type 1 de 
découverte récente que dans la population générale [11]. De même, une étude prospective a montré une relation chronologique entre marqueurs d'infection par entérovirus et séroconversion pour les anticorps anticellules d'îlot chez des enfants apparentés du premier degré à des patients atteints de diabète de type 1 [12]. Des données analogues ont récemment été obtenues en ce qui concerne les infections à rotavirus [13].

Les virus pourraient intervenir dans le déclenchement ou la modulation du processus auto-immun par différents mécanismes possiblement intriqués.

Un effet cytopathogène direct, responsable de la libération d'autoantigènes insulaires séquestrés (ou "cryptiques») pourrait expliquer I'activation de clones $T$ quiescents, n'ayant pas subi de sélection thymique négative, et le développement de l'auto-immunité (figure 1). De fait, certaines souches de virus coxsackie
B peuvent infecter les cellules $\beta$ humaines et induire des anomalies fonctionnelles et/ou la mort cellulaire [14]. D'autres virus (par exemple le virus de la rubéole et le cytomégalovirus) ont un tropisme pour les cellules $\beta$. Ce mécanisme pourrait aussi bien déclencher la maladie autoimmune que participer à la diversification et à l'amplification d'une réponse auto-immune déjà engagée. L'expression à la surface des cellules $\beta$ d'antigènes viraux peut également déclencher la réponse lymphocytaire $T$ comme dans le modèle des souris transgéniques pour le virus SV40 [15].

Une autre hypothèse fait intervenir le concept séduisant de mimétisme moléculaire [16]. Une réponse immunitaire normale vis-à-vis d'un antigène environnemental deviendrait pathogène pour les cellules $\beta$ du fait de l'existence d'homologies de structure avec un antigène insulaire (figure 2).

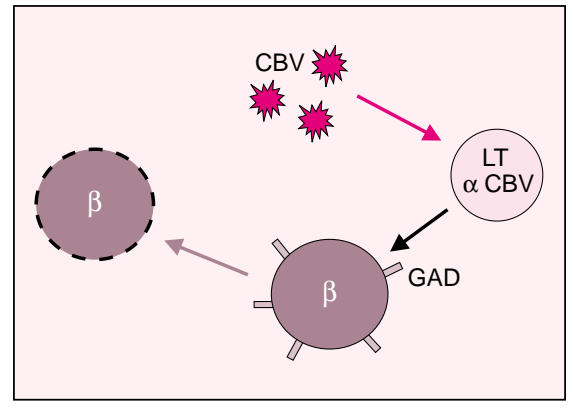

Figure 2. Mimétisme moléculaire. L'infection par le virus (ici, le virus coxsackie, CBV) déclenche une réponse immunitaire antivirale normale impliquant l'activation de lymphocytes $T(L T)$ spécifiques. Des homologies de séquence entre antigènes viraux et antigènes de la cellule $\beta$ (ici la décarboxylase de l'acide glutamique, GAD) sont responsables $d^{\prime} u n e$ réaction croisée conduisant à la destruction des cellules $\beta$.

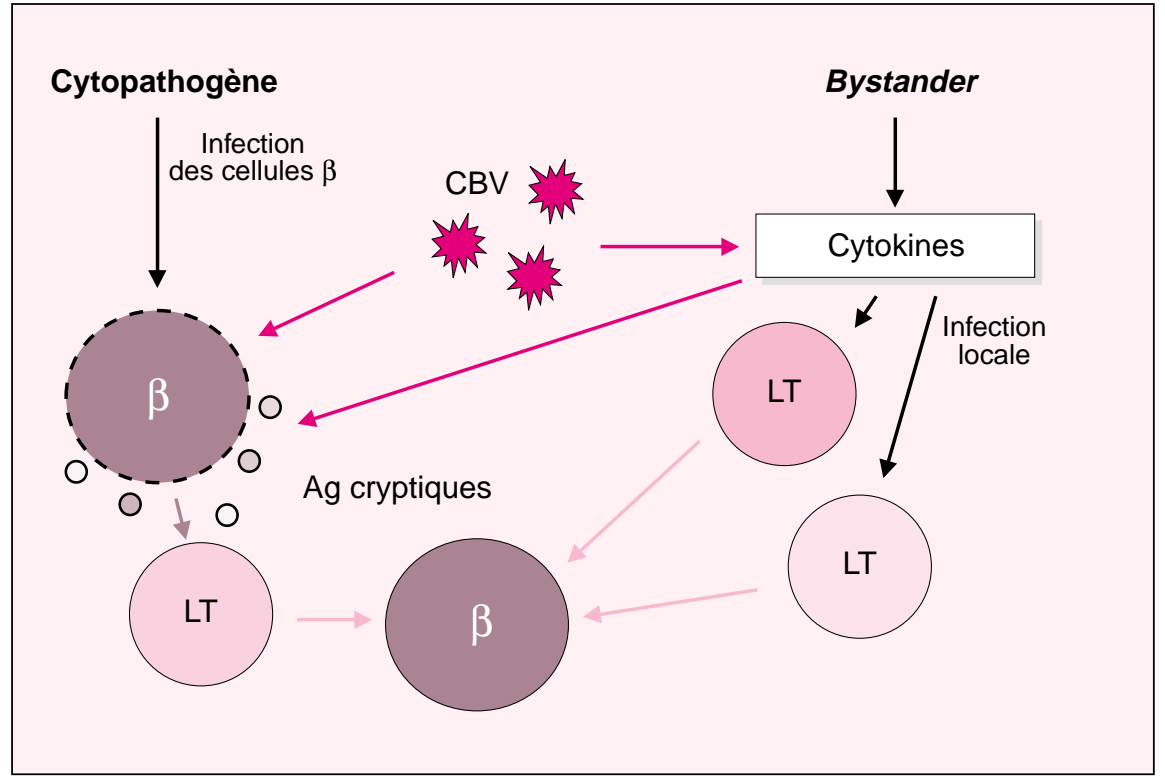

Figure 1. Effet cytopathogène et effet bystander. Un effet cytopathogène direct du virus (ici le virus coxsackie, CBV) sur la cellule $\beta(\beta)$ induit la libération d'antigènes insulaires normalement séquestrés ( $\mathrm{Ag}$ cryptiques). Cette libération conduit à l'activation d'une réponse immunitaire normale impliquant l'activation de lymphocytes $T(L T)$ autoréactifs spécifiques de ces antigènes, n'ayant pas subi de sélection thymique négative et antérieurement quiescents. Cet effet pourrait engendrer le processus auto-immune ou participer à son extension. L'infection locale par un virus à tropisme pancréatique conduit également à la production de cytokines qui fournissent un signal d'activation "bystander" à des lymphocytes $T$ autoréactifs quiescents de spécificités variées. Les cytokines peuvent également, par un effet cytopathogène direct, induire la libération d'antigènes cryptiques par les cellules $\beta$. Ces mécanismes peuvent participer à la diversification et à l'extension $d u$ processus auto-immune.
Cette hypothèse a reçu un support expérimental dans un modèle de souris transgénique. Dans ce modèle, I'expression constitutive d'une glycoprotéine virale dans les cellules $\beta$, d'une part, et d'un récepteur T dirigé contre cet antigène sur les lymphocytes $\mathrm{T}$, d'autre part, ne conduit pas à la destruction des cellules $\beta$. En revanche, l'infection des animaux par le virus déclenche un diabète " autoimmun» du fait de l'activation normale des lymphocytes T [17]. De multiples homologies de séquence entre des auto-antigènes de la cellule $\beta$ et des virus ont été rapportées (Tableau I). L'exemple le plus étudié a été celui de l'homologie entre la GAD et la protéine P2-C du virus coxsackie B. Une réactivité lymphocytaire $T$ croisée a été démontrée chez la souris, mais reste très controversée chez l'homme et, à l'heure actuelle, aucun argument formel ne permet d'impliquer le mimétisme moléculaire dans le déclenchement de la maladie auto-immune [16].

Un mécanisme d'activation non spécifique (bystander*) a été récemment

* Phénomène de toxicité de proximité décrit sous le terme anglais de bystander effect. 
Tableau I. Exemples de mimétisme moléculaire entre agents de l'environnement et antigènes exprimés par les cellules $\beta$ pancréatiques (liste non exhaustive).

\section{Agent lié à l'environnement}

Virus coxsackie B

Rubivirus

Cytomégalovirus

P73 rétrovirus $A$

Rotavirus

k-caséine

Albumine bovine

\section{Antigène insulaire}

\section{GAD}

Protéine de $52 \mathrm{kDa}$

Protéine de 38 kDa

Insuline

IA2, GAD

IA2

P69 (ABBOS)

CMV: cytomégalovirus; BSA: albumine sérique d'origine bovine.

démontré dans un modèle de souris transgénique exprimant un clone $\mathrm{T}$ diabétogène, dirigé contre un antigène des cellules $\beta$ différent de la GAD (figure 1). Chez ces souris, l'infection par le virus coxsackie B 4 induit un diabète, dont la survenue est donc sans rapport avec le mimétisme moléculaire entre le virus coxsackie B et la GAD. Le tropisme pancréatique (en l'occurrence pour le pancréas exocrine) du virus est une condition nécessaire au déclenchement de la maladie. La libération d'antigènes cryptiques et l'activation locale de la réponse autoimmune par des cytokines pro-inflammatoires pourraient être impliquées [18]. Chez la souris NOD, l'infection par le virus coxsackie B 4 (CBV 4) de la souris NOD n'accélère la survenue du diabète clinique que lorsque l'insulite est déjà étendue. Ces données suggèrent que l'infection par le virus coxsackie 4 ne peut déclencher la maladie mais que l'infection locale peut amplifier le processus auto-immun [19]. Elles montrent également qu'un même agent lié à l'environnement peut avoir des effets variables sur l'évolution de l'auto-immunité anti-cellules $\beta$ selon la chronologie de son intervention. Chez I'homme, l'étude castémoin la plus récente confirme que les infections néonatales sont associées à une augmentation du risque de diabète de type 1 avant l'âge de 15 ans (avec un risque relatif de 1,6), sans qu'il soit possible d'incriminer un agent infectieux en particulier [20].

La mise en jeu d'une activation polyclonale massive de lymphocytes T, incluant des lymphocytes T autoréactifs, par un superantigène a également été évoquée chez I'homme (figure 3). Chez deux patients décédés peu après le diagnostic de diabète de type 1 , une sur-représentation de la sousfamille lymphocytaire $T \vee \beta /$ a été observée au sein de l'insulite [21]. Cela suggère un autre mécanisme non spécifique d'amplification de la maladie auto-immune anti-cellule $\beta$. Les agents infectieux en cause restent inconnus. En effet, les travaux suggérant l'implication d'un rétrovirus (HERV) ont été infirmés par plusieurs groupes (m/s 1997, $\left.n^{\circ} 11, p .1362\right)$. Chez la souris NOD, on n'observe d'ailleurs pas de biais du répertoire lymphocytaire $\mathrm{T}$ dans I'insulite. Les rétrovirus sont en théorie susceptibles d'induire une réponse auto-immune par de nombreux autres mécanismes: expression au niveau de la cellule cible d'antigènes viraux, mimétisme moléculaire (réactivité croisée des auto-anticorps anti-insuline avec I'antigène rétroviral p73), activation de l'expression d'auto-antigènes, effet cytopathogène.

L'environnement peut également exercer des effets protecteurs vis-à-vis du développement du diabète de type 1. Chez la souris NOD, les infections précoces par divers virus (chorioméningite lymphocytaire, lactico-deshydrogénase, hépatite) réduisent l'incidence du diabète. A l'inverse, la prévalence du diabète est accrue chez les souris NOD et les rats $\mathrm{BB}$ élevés en conditions stériles [1]. Chez I'homme, les gradients Nord-Sud d'incidence du diabète de type 1 et de I'hépatite A sont inverses, ce qui suggère également un rôle protecteur de certains agents infectieux. D'autres agents infectieux, en particulier les mycobactéries, pourraient avoir le même effet protecteur. Chez la souris $N O D$ et le rat $B B$, à condition d'être précoce, I'injection d'adjuvant complet de Freund - émulsion contenant des mycobactéries confère une protection contre le diabète [22]. Ces résultats ont été reproduits par vaccination par le BCG. Le mécanisme de cette protection, qui est transférable à des animaux naîfs, $\mathrm{n}^{\prime}$ est pas spécifique et fait probablement intervenir une immunomodulation par des cytokines suppressives. Chez I'homme, les données épidémiologiques $\mathrm{n}^{\prime}$ indiquent pas le rôle protecteur de la vaccination par le BCG [23].

\section{Le rôle des facteurs diététiques}

Chez les modèles animaux, diverses interventions diététiques peuvent moduler I'histoire naturelle de la maladie. Chez I'homme, les premiers travaux suggérant l'influence de facteurs nutritionnels sur l'incidence du diabète de type 1 ont montré un lien entre la consommation d'aliments riches en nitrosamines (composés toxiques pour les cellules $\beta$ ) ou en nitrites, ou de nitrates contenus dans l'eau de boisson [24].

Depuis, sur la base d'une corrélation positive entre consommation de lait de vache et prévalence du diabète de type 1, un intérêt particulier a été porté au lien potentiel entre modalités d'allaitement dans la petite enfance et survenue d'un diabète de type 1. Cette association a conduit à deux hypothèses: d'une part, un allaitement maternel suffisamment long (3 à 18 mois) pourrait protéger contre la survenue ultérieure d'un diabète de type 1 ; d'autre part, l'introduction précoce de protéines du lait de vache, chez des sujets à risque génétique de diabète de type 1 , pourrait constituer un facteur de risque. Deux méta-analyses d'études rétrospectives ont confirmé une augmentation modeste (risque relatif de $1,5)$ du risque de diabète de type 1 chez les enfants non allaités par leur mère ou de façon brève [25]. Le risque serait plus élevé chez les sujets porteurs des haplotypes HLA de sus- 


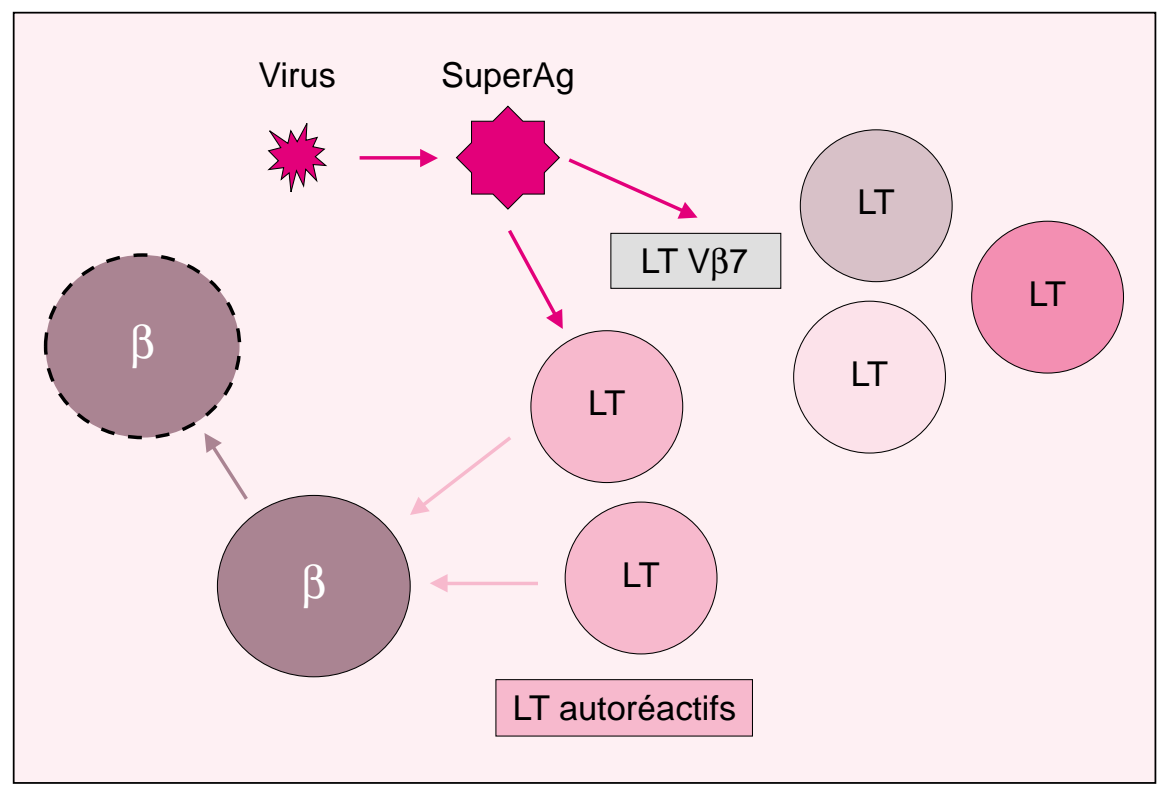

Figure 3. Activation par un superantigène. Les superantigènes (SuperAg) produits par les virus induisent l'activation polyclonale d'une sous-famille lymphocytaire $T$ (ici, V $\beta 7$ ), comportant des lymphocytes $T(L T)$ autoréactifs dirigés contre les cellules $\beta(\beta)$.

ceptibilité au diabète de type 1 [26]. L'analyse intermédiaire d'un essai de prévention du diabète de type 1 par exclusion des protéines de lait de vache dans les 6 premiers mois de vie (essai TRIGR chez les enfants à haut risque génétique) suggère une réduction d'un facteur 4 de la fréquence d'apparition des auto-anticorps anticellules $\beta$ chez les enfants ayant bénéficié de I'allaitement maternel [25]. Cependant, trois études n'ont pas montré d'association entre I'apparition de marqueurs d'autoimmunité anti-cellules $\beta$ et les modalités d'allaitement chez les enfants à haut risque (apparentés du $1^{\text {er }}$ degré d'un sujet diabétique de type 1) [2729].

La physiopathologie de cette association reste très floue. La composition protéique du lait de vache diffère de celle du lait humain par sa plus forte concentration protéique (notamment en caséine), par la présence de $\beta$-lactoglobuline, et par les séquences d'acides aminés de l'albumine et de I'insuline. L'étude de la réponse immunitaire vis-à-vis des protéines du lait de vache chez les sujets présentant un diabète de type 1 récent n'a pas apporté d'argument décisif. La présence chez ces patients d'anticorps anti-albumine (BSA) et anti- $\beta$ - caséine. Chez le rat BB, cependant, I'effet aggravant du régime standard est dû à des constituants végétaux et non aux protéines du lait de vache [28].

\section{Conclusions}

L'implication de nombreux autres facteurs d'environnement a été suggérée (voir revue dans $[24,25])$. Dans I'étude EURODIAB par exemple, un environnement plus froid (ou un ensoleillement réduit?) est associé à un risque accru de diabète de type 1 , une carence relative en vitamine $D$ également [20]. Cela est peut-être à rapprocher des puissants effets protecteurs de la vitamine $D$ et de ses analogues dans le modèle de la souris NOD. En revanche, I'incidence du diabète de type 1 n'est pas modifiée par le calendrier vaccinal (type et âge des vaccinations) $[20,30]$.

La multiplicité des agents incriminés, qui constituent autant de facteurs confondants, souligne la difficulté de I'identification, sur la base des seules données épidémiologiques, des facteurs modulant le risque de diabète de type 1 . Aucun des facteurs d'environnement suspectés à ce jour ne semble jouer un rôle majeur, en particulier dans le déclenchement de la maladie. Les données disponibles sont en faveur de l'intervention de plusieurs facteurs, certains favorisant, d'autres limitant l'agressivité de I'auto-immunité anti-cellules $\beta$, éventuellement différents d'un individu à I'autre en fonction du terrain génétique, et dont les effets peuvent également varier selon le moment de leur intervention dans l'histoire naturelle du diabète de type 1 . L'identification de ces facteurs pourrait conduire à des interventions thérapeutiques destinées à limiter la destruction des cellules $\beta$ à un stade infraclinique chez les patients porteurs de marqueurs d'auto-immunité prédictifs. Une meilleure compréhension des phases initiales de la maladie auto-immune conduisant au diabète de type 1 est nécessaire, reposant en particulier sur des études prospectives chez les sujets à risque génétique issus de la population générale. Si l'intervention de facteurs initiateurs était confirmée, elle conduirait à envisager une véritable prévention primaire du diabète de type 1 


\section{RÉFÉRENCES}

1. Bach JF. Insulin-dependent diabetes mellitus as an autoimmune disease. Endocrine Rev $1994 ; 15:$ 516-42.

2. Kyvik KO, Green A, Beck-Nielsen $\mathrm{H}$. Concordance rates of insulin dependent diabetes mellitus: a population based study of young Danish twins. Br Med J 1995; 311 . 913-7.

3. EURODIAB ACE Study Group. Variations and trends in incidence of childhood diabetes in Europe. Lancet 2000; 355 : 873-6.

4. Bodansky HJ, Staines A, Stephenson C, Haigh D, Cartwright R. Evidence for an environmental effect in the aetiology of insulin-dependent diabetes in a transmigratory population. Br Med J 1992; 304 : 1020

5. Ziegler AG, Hummel $M$, Schenker $M$ Bonifacio E. Autoantibody appearance and risk for development of childhood diabetes in offspring of parents with type 1 diabetes The 2-year analysis of the german BABYDIAB study. Diabetes 1999; 48: 460-8.

6. Gardner SG, Gale EAM, Williams AJK Gillespie KM, Lawrence KE, Bottazzo GF Bingley PJ. Progression to diabetes in relatives with islet autoantibodies. Is it inevitable? Diabetes Care 1999; 22 : 2049-54.

7. Dahlquist G, Kallen B. Time-space clustering of time at birth in childhood onset diabetes. Diabetes Care 1996; 19: 328-32.

8. Hober D, Andréolotti L, Hober C, et al. Entérovirus et diabète de type 1. Med $S_{C i}$ 1998; 14 : 398-403.

9. Dahlquist GG, Boman JE, Juto P. Enteroviral RNA and IgM antibodies in early pregnancy and risk for childhood-onset IDDM in offspring. Diabetes Care 1999; 22: 3645 .

10. Viskari HR, Koskela $P$ Lönnrot $M$, Luonuansuu S, Reunanen A, Baer $M$, Hyöty $H$. Can enterovirus infections explain the increasing incidence of type 1 diabetes? Diabetes Care 2000 : 23: 414-5.

11. Clements CB, Galbraith D, Taylor KW. Coxsackie $B$ virus infection and onset of childhood diabetes. Lancet 1995; 346: 221 3 .

12. Lönrot M, Korpela K, Knip M et al. Enterovirus infection as a risk factor for $\beta$-cell autoimmunity in a prospectively observed birth cohort. The Finnish Diabetes Prediction and Prevention Study. Diabetes 2000 49: 1314-8.

13. Honneyman MC, Coulson BS, Stone NL, et al. Association between rotavirus infection and pancreatic islet autoimmunity in children at risk of developing type 1 diabetes. Diabetes 2000 ; 49: 1319-24.

14. Roivanen M, Rasilanen S, Ylipaasto $P$, et al. Mechanisms of coxsackievirus-induced damage to human pancreatic $\beta$-cells. J Clin Endocrinol Metab 2000; 85: 432-40.
15. Adams TE, Alpert S, Hanahn D. Non tolerance and autoantibodies to a transgenic self antigen expressed in pancreatic betacells. Nature $1988 ; 325$ : 223-8.

16. Albert LJ, Inman RD. Molecular mimicry and autoimmunity. N Engl J Med 1999; 341: 2068-74.

17. Ohashi PS, Oehen S, Buerki K, et al. Ablation of «tolerance» and induction of diabetes by virus infection in viral antigen transgenic mice. Cell 1991; 65: 305-17.

18. Horwitz MS, Bradley LM, Harbertson J, Krahl T, Lee J, Sarvetnick N. Diabetes induced by coxsackie virus : initiation by bystander damage and not molecumar mimicry. Nat Med 1998; $4: 781-5$.

19. Serreze DV, Ottendorfer EW, Ellis TM, Gauntt CJ, Atkinson MA. Acceleration of type 1 diabetes by a coxsackievirus infection requires a preexisting critical mass of autoreactive T-cells in pancreatic islets. Diabetes 2000; 49: 708-11.

20. The EURODIAB Substudy 2 Study Group. Infections and vaccinations as risk factors for childhood type 1 (insulin-dependent) diabetes mellitus: a multicentre case-control investigation. Diabetologia 2000; 43: 47-53.

21. Conrad B, Weidmann E, Trucco G, Rudert WA, Behboo R, Ricordi C, Rodriguez-Rilo H, Finegold 'D, Trucco $M$. Evidence for superantigen involvment in insulin-dependent diabetes mellitus aetiology. Nature $1994 ; 371: 351-5$

22. Sadelain MW, Qin H, Sumoski W, et al. Prevention of diabetes in the BB rat by early immunotherapy using Freund's adjuvant. J Autoimmun 1990; 3:671-80.

23. Dahlquist G, Gothefors L. The cumulative incidence of childhood diabetes mellitus in Sweden unaffected by BCG-vaccination. Diabetologia 1995; 38: 873-4.

24. Dahlquist G. The aetiology of type 1 diabetes: an epidemiological perpective. Acta Paediatr 1998; 425 (suppl) : 5-10.

25. Knip M, Akerblom HK. Environmental factors in the pathogenesis of type 1 diabetes mellitus. Exp Clin Endocrinol Diabetes 1999; 107 (suppl. 3) : S93-100.

26. Virtanen SM, Laara E, Hypponen E, Reijonen H, Rasanen L, Aro A, Knip M, Ilonen J, Akerblom HK, and the chilhood diabetes in Finland study group. Cow's milk consumption, HLA-DQB1 genotype, and type 1 diabetes. Diabetes $2000 ; 49$ : 912-7.

27. Couper JJ, Steele C, Beresford S, Powell T, McCaul K, Pollard A, Gellert S, Tait B, Harrison LC, Colman PG. Lack of association between duration of breast-feeding or introduction of cow's milk and development of islet autoimmunity. Diabetes 1999; 48 : 2145-9.

28. Harrison LC, Honeyman MC. Cow's milk and type 1 diabetes. The real debate is about mucosal immune function. Diabetes $1999 ; 48: 1501-7$.

29. Hummel M, Fuchtenbusch M, Schenker $M$, Ziegler AG. No major association of breast-feeding, vaccinations, and chilhood viral diseases with early autoimmunity in the German BABYDIAB study. Diabetes Care $2000 ; 23: 969-74$.

30. Graves PM, Barriga KJ Norris JM, Hoffman MR, $Y u$ L, Eisenbarth GS, Rewers $M$. Lack of association between early childhood immunizations and $\beta$-cell autoimmunity. Diabetes Care 1999; 22: 1694-7.

\section{$\mathbf{m} / \mathbf{s} 2000$ \\ Summary}

\section{Type 1 diabetes} and environment

Type 1 diabetes is a chronic autoimmune disease directed towards the pancreatic beta-cells and resulting in absolute insulin deficiency. Its long preclinical stage is characterized by specific markers of ongoing autoimmune disease. Type 1 diabetes occurs in genetically predisposed individuals. However, in monozygotic twins, the concordance rate for the disease is only partial. This, and the rapid increase in the incidence of type 1 diabetes over the recent years suggest that environmental factors modulate the onset or the progression of the autoimmune process. Many putative agents, particularly viruses and diet factors, have been suspected, mainly on the basis of epidemiological studies. Among viruses, enteroviruses, particularly coxsackie, may either initiate the disease by materno-fœtal infection or enhance autoimmune disease through bystander activation. A short duration of breast-feeding and the early introduction of cow's milk have been also epidemiologically involved in the onset of type 1 diabetes, but the mechanisms of this association remain elusive. The identification of environmental promoters of type 1 diabetes is a difficult task because various agents may be implicated, and some may be also protective as shown in experimental models. Prospective studies in at-risk individuals are needed to identify common environmental triggers of type 1 diabetes before primary prevention may be envisaged. 